Quim. Nova, Vol. 29, No. 2, 194-199, 2006

\title{
PREPARAÇÃO E CARACTERIZAÇÃo DE SUBSTRATOS SERS ATIVOS: UM ESTUDO DA ADSORÇÃO DO CRISTAL VIOLETA SOBRE NANOPARTÍCULAS DE PRATA
}

\author{
Henrique de Santana*e Dimas A. M. Zaia \\ Departamento de Química, Universidade Estadual de Londrina, CP 6001, 86051-990 Londrina - PR \\ Paola Corio \\ Instituto de Química, Universidade de São Paulo, CP 26077, 05513-970 São Paulo - SP \\ Fady El Haber e Guy Louarn \\ Institut des Matériaux Jean Rouxel, B. P. 32229, 44322 Nantes cedex 3, França
}

Recebido em 26/7/04; aceito em 30/6/05; publicado na web em 20/1/06

\begin{abstract}
PREPARATION AND CHARACTERIZATION OF SERS-ACTIVE SUBSTRATES: A STUDY OF THE CRYSTAL VIOLET ADSORPTION ON SILVER NANOPARTICLES. The structural characterization of molecules used in the sterilization of blood for transfusions, such as crystal violet (CV), is relevant for understanding the action of these prophylactic drugs. The characterization is feasible by surface enhanced resonance Raman spectroscopy (SERRS) of CV in solution or on surfaces. The limit of detection of CV by SERRS, in the presence of colloidal particles, using $514.5 \mathrm{~nm}$ as excitation radiation, was found to be around $1 \mathrm{ppb}$. The characterization of $\mathrm{CV}$ was also made by SERS, by using different active-particles-containing substrates, proving the versatility of this technique for the study of such structures. The results suggest that the controlled production of highly efficient SERS-active substrates may allow qualitative and quantitative analysis, with high sensitivity, with potential applications in medical and environmental fields.
\end{abstract}

Keywords: silver sol; crystal violet; surface enhanced resonance Raman spectroscopy (SERRS).

\section{INTRODUÇÃO}

Certas parasitoses estão entre as mais prevalentes doenças do mundo, atingindo milhões de pessoas. A prevenção destas doenças depende de medidas sanitárias, assim como do emprego de fármacos antiparasitários eficazes e seguros. Um importante exemplo é a doença de Chagas, que afeta aproximadamente 16-18 milhões de pessoas, principalmente nas Américas do Sul e Central, constituindo um sério problema de saúde pública. Desde a descoberta da doença pelo médico sanitarista Carlos Chagas, em 1909, até os dias atuais, foram realizadas inúmeras tentativas de tratamento, sem que fosse obtido um medicamento totalmente eficaz. Para eliminação do parasita do sangue contaminado em bancos de sangue, a única substância utilizada como agente quimioprofilático é o cristal violeta $(\mathrm{CV})$, também conhecido como violeta de genciana. Quando essa substância é adicionada ao sangue estocado na concentração de $250 \mu \mathrm{g} / \mathrm{mL}$, promove eliminação segura das formas infectantes. Porém, seu uso também é limitado pelos efeitos colaterais e pela cor transferida à pele e à urina dos pacientes transfundidos ${ }^{1}$.

Para desenvolvimento de fármacos mais seletivos e eficazes é cada vez mais importante a seleção do alvo terapêutico, o que permite uma busca racional por agentes que provoquem a resposta desejada e específica para o controle da doença. A forma como o parasita causador da doença de Chagas (Trypanosoma cruzi) relaciona-se com a molécula de cristal violeta demanda um estudo visando a identificação desta interação. Conhecer os mecanismos envolvidos nessa relação favorece o desenvolvimento de novas terapias para combater a doença. No caso do cristal violeta ou violeta de genciana suas reações são pouco estudadas, demonstrando a complexidade deste composto da classe dos corantes trifenilmetânicos ${ }^{2,3}$.

*e-mail: hensan@uel.br
Freqüentemente são utilizados para análise dos corantes os métodos cromatográficos ${ }^{4,5}$, com detecção por colorimetria e, às vezes, por fluorimetria. Por consequiência, uma caracterização estrutural destas moléculas não é obtida. Somente as técnicas espectroscópicas (RMN, espectroscopias óticas) permitem este tipo de caracterização ${ }^{6}$, mas sua baixa sensibilidade as tornam inadequadas para detecção na saída da coluna (destacamos, porém, a utilização da espectrofotometria no infravermelho para cromatografia em fase gasosa).

A espectroscopia Raman ${ }^{7}$ apresenta várias vantagens no ambiente em que se pretende trabalhar e pode resolver eventuais problemas de tempo de análise. Essa técnica requer pouca preparação de amostras e possibilita a análise de espécies em solução aquosa (uma vez que a água tem pouca interferência). Além disso, permite a identificação e a quantificação de espécies químicas, uma vez que o conjunto dos modos vibracionais das amostras está univocamente relacionado à sua identidade química.

Por outro lado, o fato de o espalhamento Raman ser um processo muito pouco eficiente - devido à sua pequena seção de choque (cerca de $10^{-30} \mathrm{~cm}^{2} \mathrm{Sr}^{-1}$ molécula $\left.{ }^{-1}\right)^{8}$ - faria da espectroscopia Raman uma técnica pouco sensível. Entretanto, a presença de superfícies metálicas nanoestruturadas apresenta a propriedade de intensificar o sinal Raman de moléculas adsorvidas por fatores da ordem de $10^{6}$ vezes. Essa intensificação, por diversas ordens de magnitude, do sinal Raman de espécies adsorvidas em superfícies metálicas especialmente preparadas é chamado de efeito Raman intensificado pela superfície, ou SERS ("Surface Enhanced Raman Scattering" $)^{9,10}$. A existência desse efeito permite o uso da espectroscopia Raman de forma muito mais abrangente, abrindo possibilidades de aplicação da espectroscopia vibracional para análise nas escalas de nano-, pico- e femtograma ${ }^{11}$. Considerando o potencial das observações experimentais, o efeito SERS se tornou uma técnica bem estabelecida, aplicável à química de superfícies 
para caracterização de adsorbatos e de transformações químicas ocorrendo em superfícies metálicas ${ }^{12}$. Do ponto de vista de análise química, os aspectos mais interessantes da técnica SERS são sensibilidade e seletividade.

Muitas aplicações interessantes referem-se ao uso do efeito SERS nas áreas médica e ambiental ${ }^{13}$. Duas de suas características tornamno bastante conveniente para ser utilizado nestes campos. Por um lado, o efeito SERS é muitas vezes acompanhado por uma forte extinção da fluorescência (característica de muitas biomoléculas e também de moléculas de interesse ambiental, como os hidrocarbonetos policíclicos aromáticos, por ex.), o que permite estender o grupo de compostos estudados por espectroscopia Raman. Além disso, a alta sensibilidade da técnica permite também que sejam obtidos espectros de moléculas em concentrações da ordem de $10^{-10} \mathrm{~mol} \mathrm{~L}^{-1}$. Por tudo isso, o efeito SERS já foi utilizado em diversos estudos de relevância biológica, como a determinação da distribuição de drogas e componentes celulares em uma célula viva ou em membranas celulares ${ }^{14,15}$.

A área de SERS tem sido uma das que mais experimentou avanços dentre as pesquisas em espectroscopia Raman nos últimos anos. Historicamente, as aplicações analíticas das técnicas Raman eram limitadas por sua relativa baixa sensibilidade, se comparada, por ex., à técnica de fluorescência. Desenvolvimentos recentes, entretanto, tiveram impacto particularmente importante no sentido de mudar essa tendência: grandes intensificações do sinal Raman foram observadas em experimentos SERS em superfícies especialmente preparadas. Dessa forma, o aperfeiçoamento das técnicas SERS possibilitou a detecção do espectro vibracional de uma única molécula. Os primeiros trabalhos a esse respeito foram relatados em 1997 ${ }^{16,17}$. Desde então, esses resultados foram confirmados por diversos grupos ${ }^{18}$. Assim, a técnica já é sensível o suficiente para competir com técnicas baseadas em fluorescência, com as quais já eram obtidos, há mais tempo, espectros de moléculas isoladas. Entretanto, considerando-se a riqueza de informações contidas em um espectro vibracional, em comparação com um espectro de fluorescência, é de se esperar que a técnica SERS se torne cada vez mais importante na área de detecção química.

Com o objetivo de se utilizar a técnica SERS em ambientes reais, muitos estudos foram feitos no sentido de se desenvolver substratos duráveis, de baixo custo, e que pudessem ser preparados de maneira reprodutível. A base é, invariavelmente, a produção de uma superfície metálica rugosa. Existe hoje uma grande variedade de substratos SERS ativos ${ }^{19}$. Pesquisadores têm conseguido selecionar determinadas arquiteturas para o substrato que estejam de acordo com suas necessidades experimentais. Através de escolhas criteriosas do metal de um substrato, do grau de oxidação da superfície, das propriedades ácido/base das superfícies, do uso de recobrimentos, e outros parâmetros, o substrato SERS ativo pode ser desenvolvido de modo a adsorver de maneira altamente seletiva compostos selecionados com estruturas químicas semelhantes.

O principal objetivo deste trabalho é apresentar possibilidades de desenvolvimento de novos substratos SERS ativos, capazes de superar as limitações dos substratos tradicionais e possuindo características como maiores fatores de intensificação, maior reprodutibilidade em sua preparação e maior especificidade.

As novas técnicas que vêm sendo desenvolvidas nessa área nos últimos anos têm permitido a obtenção de substratos mais duráveis. Os aperfeiçoamentos propostos nos processos de preparação dos substratos conduziram a ganhos importantes nas possibilidades de determinação quantitativa de espécies químicas ${ }^{20}$. Informações sobre a cobertura das partículas, tamanho e espaço entre as partículas das superfícies preparadas podem ser obtidas por técnicas de microscopia como AFM e TEM - e este trabalho oferece exemplos disso. A utilidade prática de um substrato é definida por uma série de características: tipo mais adequado em cada caso específico, grupos funcionais e polímeros viáveis para associação com o metal visando melhorias nas propriedades, estabilidade, reprodutibilidade e propriedades ópticas.

Dentre os substratos mais utilizados, os colóides têm sido estudados devido a suas propriedades ópticas particulares. Eles se tornaram populares, como substratos ativos para o efeito SERS, desde a obtenção do espectro SERS da piridina em colóide de prata, em 1979, por Creighton et al. ${ }^{21}$. Os colóides apresentam numerosas vantagens: sua preparação é relativamente simples, a superfície ativa pode ser renovada continuamente por agitação da suspensão, o tamanho e a forma de partículas podem ser medidas facilmente e podem ser caracterizadas pelo espectro de absorção UV-Vis e, por último, há a possibilidade de preparação de filmes de colóides sobre diferentes substratos.

Neste trabalho, demonstramos o grande potencial da técnica SERRS em estudos realizados em diferentes substratos SERS ativos preparados por métodos simples, a saber: soluções de prata coloidal e nanoestruturas de prata depositadas sobre suportes estacionários. Para isso, apresentamos o estudo do CV por meio de uma variedade de técnicas de espectroscopia Raman, tais como Raman normal, Raman ressonante, Raman intensificada pela superfície (SERS), e ainda Raman ressonante intensificado pela superfície (SERRS). A relação entre as características morfológicas dos substratos e sua atividade SERS também foi estudada por meio de técnicas de microscopia de força atômica e microscopia eletrônica de varredura.

\section{PARTE EXPERIMENTAL}

\section{Instrumentação}

Os espectros Raman foram obtidos usando um espectrômetro Jobin Yvon NT64000, utilizando lasers de íons argônio e kriptônio da Spectra Physics (radiações excitantes em 514,5 e 676,4 nm, respectivamente). Um microscópio Olympus foi utilizado para focalizar a radiação incidente sobre as amostras e para coletar a radiação espalhada. As medidas por Microscopia Eletrônica por Transmissão (TEM) foram obtidas em um Instrumento HF 2000 com um feixe de elétrons emitido a $200 \mathrm{kV}$, depois de se colocar uma gota de amostra dos colóides em um "grid" de carbono-Cu. As análises de Microscopia Eletrônica de Varredura (SEM) foram realizadas em equipamento JEOL, 6400F, após a metalização da amostra. As imagens de Microscopia de Força Atômica (AFM) foram obtidas através do equipamento Nanoscope III, da Digital Instruments, em modo de não contato.

\section{Preparação dos colóides de prata}

A solução de prata coloidal foi preparada de acordo com o procedimento de Lee-Meisel ${ }^{22}$, descrito a seguir. Antes da preparação toda a vidraria foi lavada com ácido nítrico $\left(\mathrm{HNO}_{3}-\mathrm{H}_{2} \mathrm{O}\right)$ na proporção $1: 1(\mathrm{v} / \mathrm{v})$. Nitrato de prata $(\approx 90 \mathrm{mg})$ foi adicionado em um erlenmeyer, envolto em papel alumínio, contendo $500 \mathrm{~mL}$ de água tridestilada e aquecido até a ebulição. Após, foi adicionada gota-agota, sob vigorosa agitação, $10 \mathrm{~mL}$ de uma solução a $1 \%$ de citrato de sódio. A solução foi deixada nestas condições por 40 min e, após retirado o aquecimento, foi mantida por 90 min com agitação contínua para resfriamento da solução. O volume foi completado para 500 $\mathrm{mL}$ com água tridestilada. Utilizou-se reagentes Aldrich e água tridestilada em todos os experimentos. Na Figura 1 é apresentado o espectro de absorção no UV-Vis da solução de prata coloidal. Observa-se que a absorção do plasmon superficial dá origem a uma banda larga, com o máximo de absorção em torno de $408 \mathrm{~nm}$. No caso deste substrato SERS ativo, teríamos intensificação do campo elétrico para 


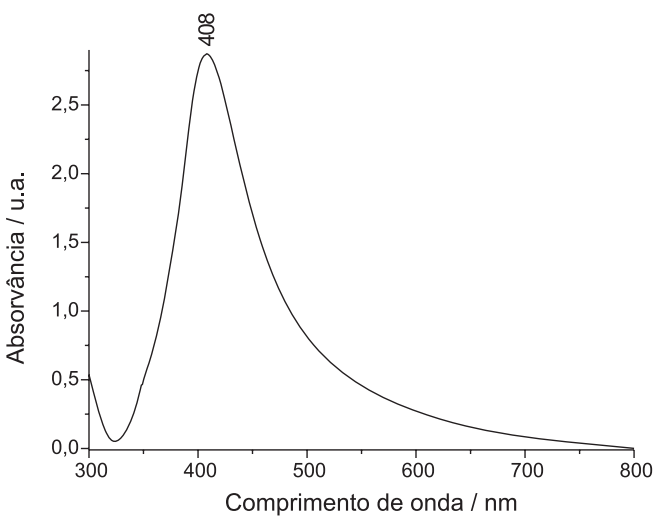

Figura 1. Espectro de eletrônico de absorção no UV-Vis da solução de prata coloidal

radiações com comprimento de onda dentro da banda de absorção do plasmon ( entre 370 e $600 \mathrm{~nm}$ ). O máximo de absorção no espectro UV-VIS de soluções coloidais está relacionado ao tamanho médio das partículas, enquanto que a largura da banda de absorção relaciona-se à dispersão das partículas ${ }^{23}$.

Durante algumas preparações do colóide de prata verificouse que o controle cuidadoso das variáveis experimentais discutidas acima é imprescindível durante a síntese. Na Figura 2(a) é apresentada a imagem de microscopia eletrônica de transmissão (TEM) de uma destas preparações, onde pode ser verificada uma agregação muito grande entre as partículas e a formação de estruturas na forma de bastonetes, devido a uma agitação pouco intensa e à utilização de água destilada. Tal colóide não apresenta atividade SERS significativa. Na Figura 2(b) é apresentada a imagem TEM da preparação do colóide de prata nas condições de síntese discutidas nos espectros de absorção da Figura 1. Neste caso, as partículas de Ag apresentam um formato esférico uniforme, com tamanho médio entre 15-20 nm, sem agregação.

Os espectros SERRS de diferentes soluções aquosas de CV em colóide de prata foram obtidos pela mistura prévia dos reagentes na proporção de 1:1, ou seja, a concentração indicada nos espectros se refere a essa diluição realizada na solução coloidal. As mesmas quantidades das soluções foram injetadas em uma cavidade de uma placa de vidro fixada ao microscópio para obtenção dos espectros. A sequiência dos espectros foi registrada após o ajuste da melhor relação sinal/ruído na primeira amostra e mantida para as demais. Os espectros foram obtidos no mesmo tempo de aquisição do sinal.

\section{Preparação dos substratos SERS ativos}

Considerando que a maioria das aplicações de caracterização por SERS são restritas a condições estacionárias, foi preparado inicialmente um substrato SERS ativo do tipo "silver islands" por "spin coating" sobre $\mathrm{SiO}_{2}$. Após a colocação de algumas gotas do colóide de prata sobre a superfície do $\mathrm{SiO}_{2}$, esta foi submetida à velocidade de 20.000 rpm durante $60 \mathrm{~s}$; em seguida, o substrato foi seco sob vácuo. Para obtenção dos espectros SERRS algumas gotas da solução de 100 ppm de CV foram adicionadas sobre o substrato, o qual foi em seguida submetido à velocidade de $20.000 \mathrm{rpm}$ durante $80 \mathrm{~s}$ e seco sob vácuo. O efeito SERRS foi confirmado pela obtenção dos espectros do cristal violeta, utilizando a radiação excitante $514,5 \mathrm{~nm}$ sobre esta superfície. Para esta preparação não pode ser estudada a topografia do substrato SERS ativo por AFM, devido à falta de contraste entre o substrato e as partículas de prata depositadas.

A deposição do colóide de Ag sobre substrato de vidro foi efetuada visando a obtenção um substrato SERS ativo com uma maior área su- a)

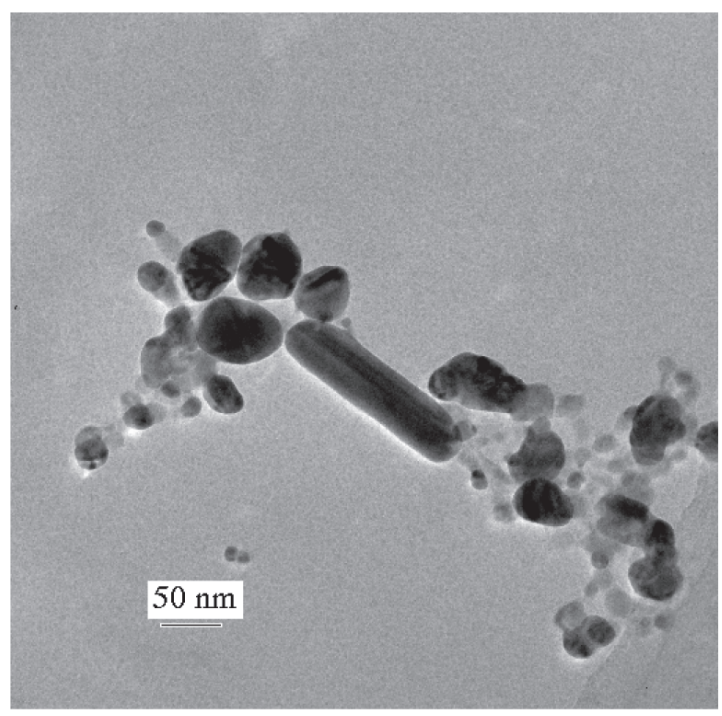

b)

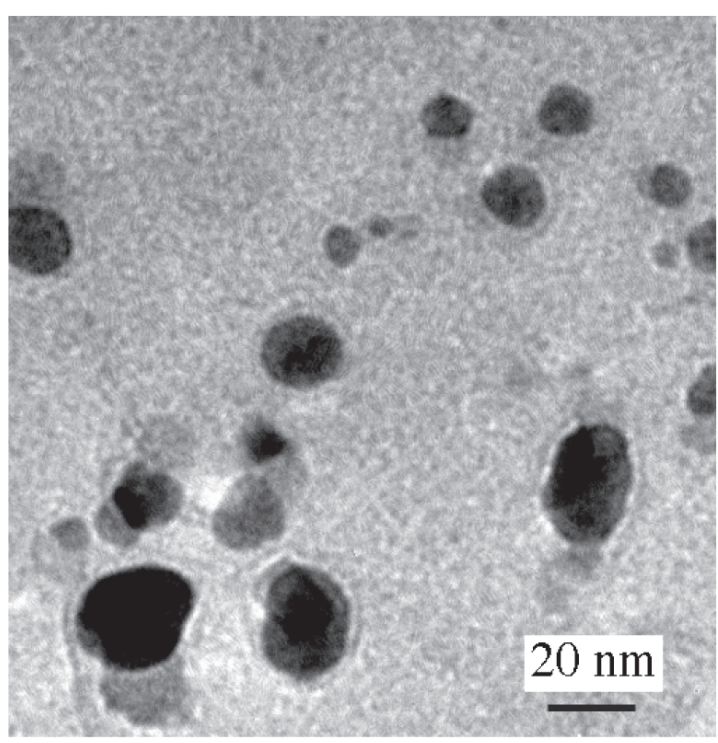

Figura 2. Imagens de microscopia eletrônica de transmissão (TEM) de (a) colóide de prata em condições de síntese que apresentaram agregação e (b) colóide de prata sem agregação (SERS ativo)

perficial. Algumas gotas do colóide de prata foram colocadas sobre a placa de vidro aquecida a $120{ }^{\circ} \mathrm{C}$ (temperatura da placa aquecedora) até a completa evaporação do solvente. Este procedimento foi escolhido por originar uma nanoestrutura com características de rugosidade próximas a superfícies formadas em sistemas eletroquímicos, após o tratamento da superfície do eletrodo metálico por ciclos de oxidação-redução ${ }^{24}$. Para obtenção dos espectros SERRS sobre esse substrato, algumas gotas da solução 3 ppm de CV foram adicionadas sobre ele, sendo retirado o excesso de solução por evaporação, através de um fluxo de $\mathrm{N}_{2}$ sobre a superfície. $\mathrm{O}$ efeito SERRS foi confirmado pela obtenção dos espectros do cristal violeta, utilizando-se a radiação excitante $514,5 \mathrm{~nm}$ sobre esta superfície. Para esta preparação foi estudada a topografia do substrato SERS ativo por técnicas de SEM e AFM.

O filme de CV sobre o vidro foi preparado pelo recobrimento com uma solução $3 \mathrm{ppm}$ de corante, sendo o excesso retirado por evaporação, através de um fluxo de $\mathrm{N}_{2}$. $\mathrm{O}$ espectro apresentado foi obtido nas mesmas condições dos espectros do CV sobre substrato de vidro com nanopartículas de prata. 


\section{RESULTADOS E DISCUSSÃO}

A Figura 3 apresenta o espectro de eletrônico de absorção do $\mathrm{CV}$ em solução aquosa, no qual observa-se uma banda intensa com máximo em $589 \mathrm{~nm}$. Representa-se, também, nessa figura as energias das linhas laser utilizadas para excitação dos espectros Raman: 514,5 e 676,4 nm. Observa-se que, enquanto a linha em $514,5 \mathrm{~nm}$ encontra-se dentro da banda de absorção do corante, a linha em 676,4 nm tem uma energia menor que a transição eletrônica em questão estando, portanto, fora da condição de ressonância. Esse efeito de ressonância sobre a intensidade de espalhamento Raman pode ser observado na Figura 4, a qual apresenta os espectros Raman convencionais de soluções aquosas de $\mathrm{CV}$ em diferentes concentrações e excitados em diferentes comprimentos de onda. O efeito Raman ressonante consiste na intensificação, por algumas ordens de grandeza, da seção de choque Raman de modos vibracionais específicos (modos vibracionais envolvidos no cromóforo). Esse efeito ocorre quando o espectro é obtido com uma energia de excitação próxima à de uma transição eletrônica permitida da molécula.

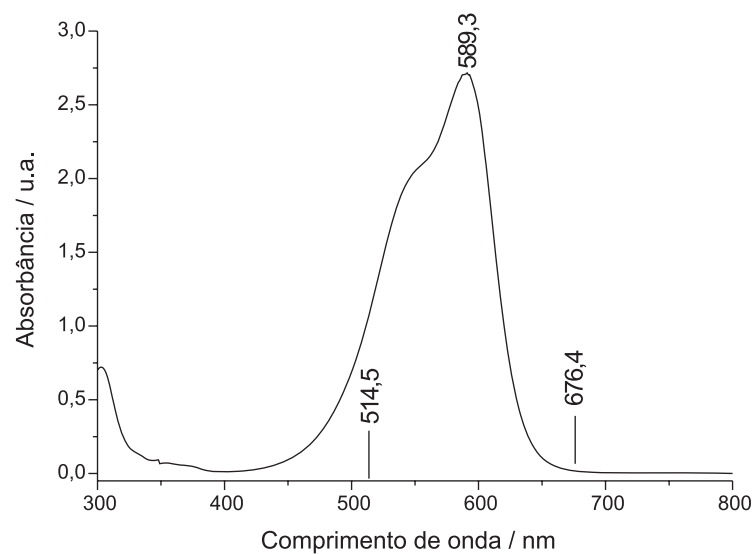

Figura 3. Espectro eletrônico de absorção no UV-Vis de uma solução aquosa de $C V$

Na Figura 4(a) é apresentado o espectro Raman do CV obtido na radiação excitante $676,4 \mathrm{~nm}$, ou seja, fora da condição de ressonância. Observa-se, nesse caso, uma forte fluorescência, o que dificulta grandemente a obtenção de um espectro vibracional de qualidade. Em condição de ressonância (radiação excitante em 514,5 nm) o espalhamento Raman torna-se mais intenso que a fluorescência e a

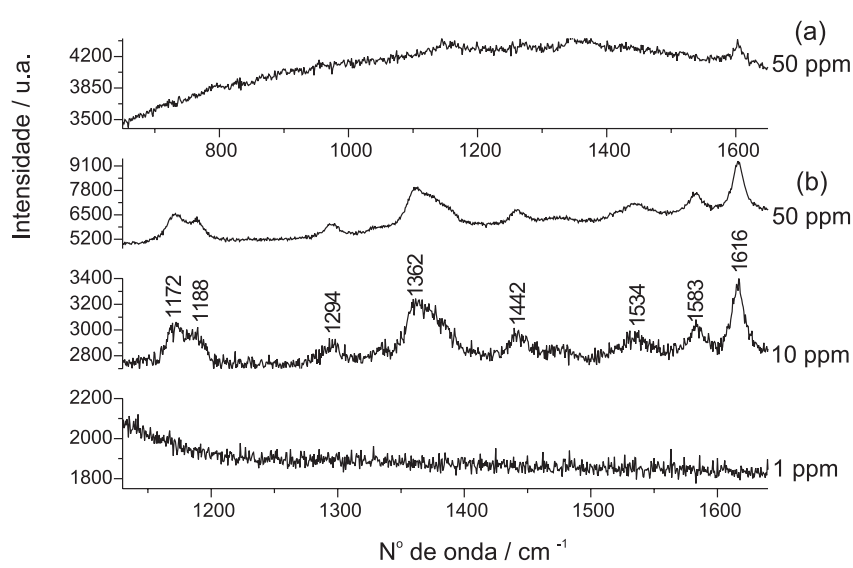

Figura 4. Espectros Raman ressonante de diferentes soluções aquosas de CV, nas concentrações indicadas e excitados em (a) 676,4 nm e (b) 514,5 nm observação dos espectros vibracionais das soluções de CV tornase possível, como apresentado na Figura 4(b). A técnica Raman ressonante é significativamente mais sensível que a espectroscopia Raman normal, sendo que a sensibilidade da técnica proporcionou observar a concentração do corante de $10 \mathrm{ppm}$.

A Figura 5 apresenta os espectros Raman de CV em solução de prata coloidal para a radiação excitante em 514,5 nm. Considerando que essa radiação encontra-se em ressonância, tanto com a absorção dos "plasmon" superficial do colóide (Figura 1), quanto com a banda de absorção do corante (Figura 3), esperamos que a intensificação SERS se combine ao efeito Raman ressonante. Dessa maneira, os dois efeitos serão multiplicativos, e teremos o chamado efeito SERRS ("Surface Enhanced Resonance Raman Scattering”). Nessas condições experimentais, a sensibilidade da técnica é consideravelmente mais alta que no caso da Figura 4, e podemos observar, com boa relação sinal/ruído, o espectro característico do CV na concentração de $1 \mathrm{ppb}$.
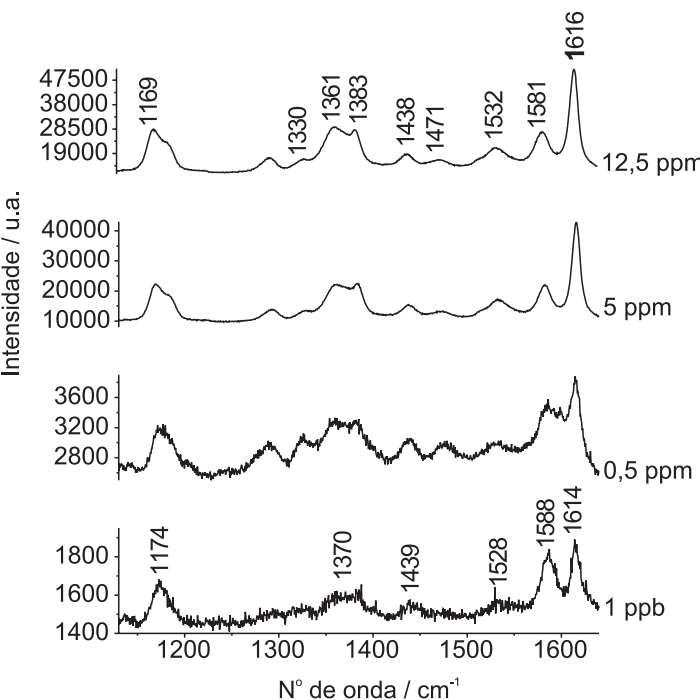

Figura 5. Espectros SERRS de diferentes soluções aquosas de CV em colóide de prata, nas concentrações indicadas e excitados em 514,5 nm

É também interessante notar que ocorrem modificações espectrais significativas, conforme varia a concentração do CV. Enquanto os espectros obtidos em concentrações maiores são semelhantes aos espectros Raman ressonante, aqueles obtidos em concentrações menores são diferentes. O sinal SERRS em baixas concentrações relaciona-se, principalmente, às moléculas que sofrem uma adsorção direta sobre a superfície do colóide de prata. Assim, as modificações espectrais estão relacionadas a interações químicas específicas entre a superfície do colóide e o adsorbato, bem como modificações de simetria relacionadas ao processo de adsorção, conforme sugerido por Li et al. ${ }^{25}$. Em baixas concentrações, o modo em 1581 desloca-se para $1588 \mathrm{~cm}^{-1}$ e aumenta significativamente sua intensidade relativa; os modos em 1169 e $1180 \mathrm{~cm}^{-1}$ coalescem em uma única banda em $1174 \mathrm{~cm}^{-1}$.

Na Figura 6(c) é apresentado o espectro do CV adsorvido sobre o substrato formado por nanopartículas de Ag depositadas sobre $\mathrm{SiO}_{2}$ (denominado $\mathrm{Ag}-\mathrm{SiO}_{2}$ ) e, para efeito de comparação, o espectro da solução $1 \mathrm{ppm}$ de $\mathrm{CV}$ em colóide de prata, Figura 6(d). São apresentados também os espectros Raman do colóide de Ag puro e do substrato de $\mathrm{SiO}_{2}$, onde são características as frequiências em 235 e 293, 493 e $924 \mathrm{~cm}^{-1}$, respectivamente. As frequiências relacionadas ao substrato de $\mathrm{SiO}_{2}$ podem ser observadas no espectro do $\mathrm{CV}$ adsorvido sobre o substrato 


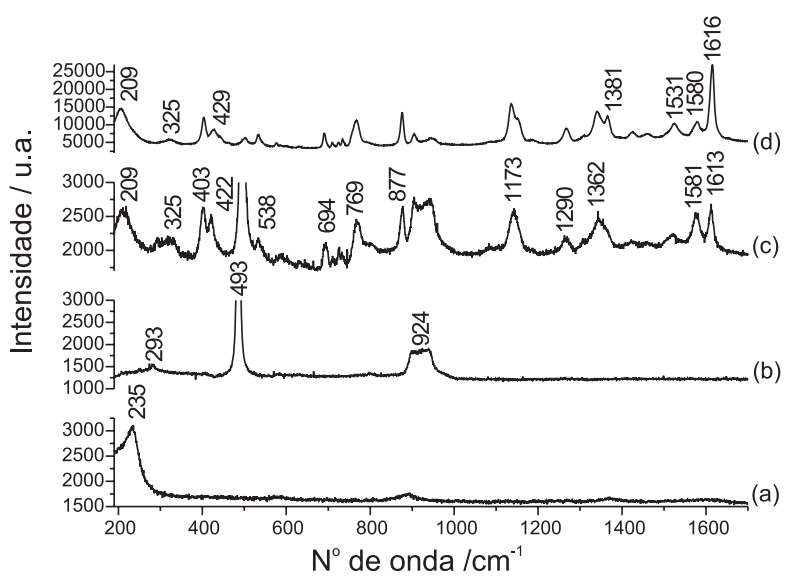

Figura 6. Espectros (a) Raman de uma solução de prata coloidal, (b) Raman do substrato de $\mathrm{SiO}_{2}$, (c) SERRS do CV adsorvido sobre nanopartículas de Ag depositadas previamente sobre $\mathrm{SiO}_{2}$ e (d) SERRS da solução de 1 ppm de CV em colóide de prata, excitados em 514,5 nm. A banda em $493 \mathrm{~cm}^{-1}$ (apresentada fora de escala) refere-se ao estiramento Si-O do substrato

$\mathrm{Ag}-\mathrm{SiO}_{2}$ (Figura 6(c)). A superfície SERS ativa $\left(\mathrm{Ag}-\mathrm{SiO}_{2}\right)$ com $\mathrm{CV}$ claramente apresentou uma intensificação do sinal Raman. O espectro SERRS do composto adsorvido sobre esta superfície é comparável ao espectro do CV na concentração de 1 ppb obtido na presença do colóide, apresentado anteriormente na Figura 5 , e difere em intensidade relativa das bandas do espectro da solução de 1 ppm de CV em colóide de prata, Figura 6(d). Não foram observadas nos espectros, na região de baixa freqüiência, diferenças entre os espectros do CV adsorvido sobre a superfície $\mathrm{Ag}-\mathrm{SiO}_{2}$ e sobre o colóide de Ag que justifiquem uma interação entre o $\mathrm{CV}$ e as partículas de $\mathrm{Ag}$, considerando que a freqüência em $209 \mathrm{~cm}^{-1}$ foi observada no espectro Raman ressonante da solução de $\mathrm{CV}^{26}$. $\mathrm{O}$ espectro do $\mathrm{CV}$ adsorvido sobre nanopartículas de Ag sobre de vidro (Figura 7b) sofreu também um significativo efeito SERS. Foram observadas as mesmas modificações nos espectros do $\mathrm{CV}$ em relação à intensificação observada sobre a superfície $\mathrm{Ag}-\mathrm{SiO}_{2}$.

Com o intuito de estudar a topografia do substrato SERS ativo $\mathrm{Ag}-\mathrm{SiO}_{2}$ antes e depois da adsorção de $\mathrm{CV}$ fez-se a tentativa de

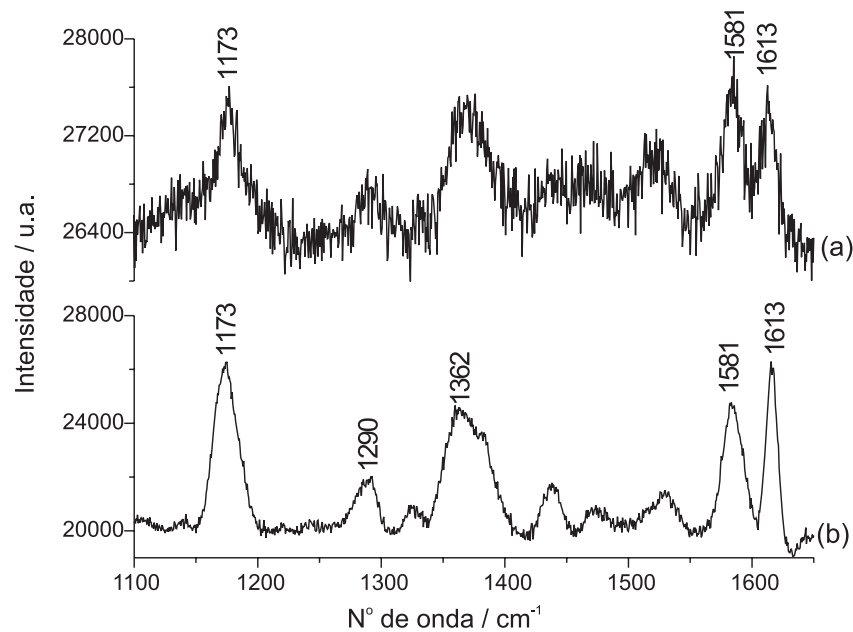

Figura 7. Espectros (a) Raman ressonante do vidro recoberto por um filme de CV e (b) SERRS do CV adsorvido sobre nanopartículas de Ag (preparadas pelo recobrimento de vidro com prata coloidal e submetido a tratamento térmico a $110{ }^{\circ} \mathrm{C}$ ) em $514,5 \mathrm{~nm}$ obter imagens AFM. Estas imagens, entretanto, não apresentaram contraste de fase, o que pode significar uma incompatibilidade entre o diâmetro das partículas de prata e o nível de detecção do equipamento ou, ainda, que os limites entre as partículas de prata e o suporte não foram suficientemente bem definidos.

A imagem de microscopia eletrônica de varredura (SEM) da placa de vidro recoberta com colóide de prata após tratamento térmico a $110{ }^{\circ} \mathrm{C}$ está mostrada na Figura 8 . A superfície das partículas de prata depositadas sobre o vidro apresenta um aspecto granular mais no fundo, com dimensões menores que $0,5 \mu \mathrm{m}$, e outra com aglomerados, na forma de bastonetes, aflorando à superfície em conjunto com partículas de prata com dimensões menores que $1 \mu \mathrm{m}$. A imagem gerada por AFM do mesmo substrato é apresentada na Figura 9, onde foi possível visualizar a topografia presente na superfície recoberta com colóide de prata. Como anteriormente observado por SEM, mas com melhor definição, a imagem AFM das partículas de prata apresentou uma aglomeração também próxima à superfície do substrato. Através da análise de seção foi possível estimar o tamanho médio das partículas de prata entre 50 e 55 nm (distância vertical), com altura média da superfície (distância horizontal) entre 170 e $250 \mathrm{~nm}$.

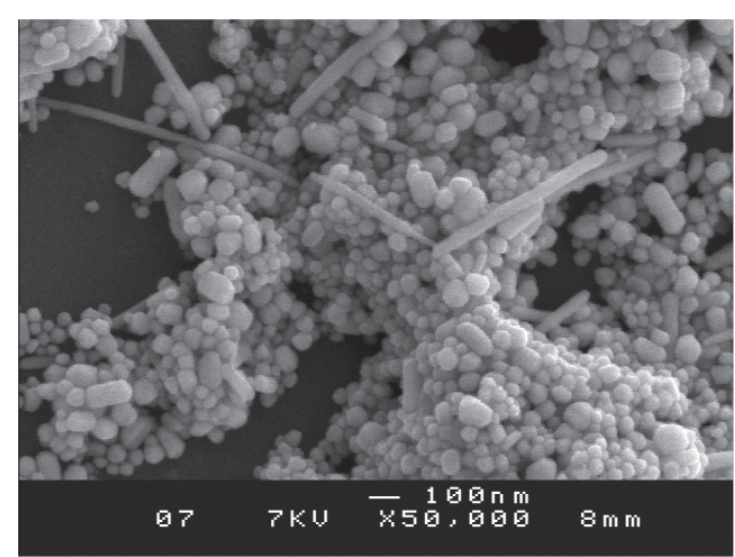

Figura 8. Imagem SEM do substrato SERS ativo de vidro recoberto com nanopartículas de prata após tratamento térmico a $110^{\circ} \mathrm{C}$

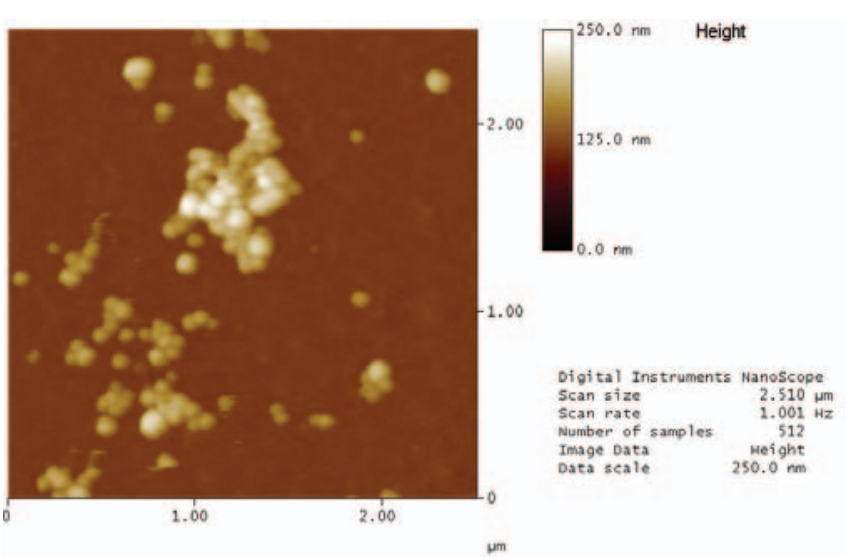

Figura 9. Imagem AFM de topografia do substrato SERS ativo de vidro recoberto com nanopartículas de prata, após tratamento térmico a $110{ }^{\circ} \mathrm{C}$. A amplificaçao lateral é de 2,51 um e a escala de altura (eixo z) é dada pela variação de tons na figura. Na imagem, a variação em altura ("Z range") é de $250 \mathrm{~nm}$, indicando que a variação da cor preta até a cor branca nas imagens corresponde a uma variação topográfica de $250 \mathrm{~nm}$ 


\section{CONCLUSÕES}

Considerando os resultados experimentais apresentados neste trabalho as seguintes conclusões podem ser destacadas: a preparação do colóide de prata, considerando as variáveis discutidas, é simples e favorece a preparação de estruturas ativas ao SERS em diferentes suportes estacionários; a sensibilidade desta técnica para determinação do CV é bastante alta. Isso demonstra a adequação da técnica SERS para estudo do corante em diferentes condições e, os diferentes substratos preparados e caracterizados nesse trabalho apresentaram boa atividade SERS, estabilidade e podem ser preparados de maneira controlada e reprodutível. Essas qualidades os tornam promissores para análises químicas qualitativas e quantitativas em alta sensibilidade, apresentando assim um grande potencial para aplicações principalmente nas áreas médicas e de interesse ambiental.

\section{AGRADECIMENTOS}

H. de Santana agradece ao programa CAPES/COFECUB pelo apoio financeiro; P. Corio, ao CNPq pela bolsa de pesquisa (CNPQ307840/2003-4) e aos projetos Fapesp (Processo: 04/07463-7), $\mathrm{CNPq}$ (Processo: 475768/2003-5) e Bolsa CNPq(307840/2003-4). Os autores agradecem à Profa. M. L. A. Temperini (LEM-IQ/USP) pelas discussões.

\section{REFERÊNCIAS}

1. http://dtr2001.saude.gov.br/svs/pub/GVE/PDF/GVE0507.pdf; http:// www.teses.usp.br/teses/disponiveis/60/60131/tde-07082003-094230/ publico/02.Introducao.pdf , acessadas em Junho 2004

2. Jonnalagadda, S. B.; Int. J. Chem. Kinet. 1992, 24, 1113.

3. Bhasikuttan, A. C.; Shastri, L. V.; Sapre, A. V.; Rama-Rao, K. V. S.; Mittal, J. P.; J. Photochem. Photobiol., A 1994, 84, 237.

4. Chen, Q.; Mou, S.; Hou, X.; Riviello, J.M.; Ni, Z.; J. Chromatogr., A 1998, 827, 73.

5. Gennaro, M. C.; Gioannini, E.; Angelino, S.; Aigotti, R.; Giacosa, D.; J. Chromatogr., A 1997, 767, 87.
6. Silverstein, R. M.; Bassler, G. C.; Morrill, T. C. Em Identificação Espectrométrica de Compostos Orgânicos; Alencastro, R. B.; Faria, R. B., trads.; $3^{a}$ ed., Guanabara Koogan: Rio de Janeiro, 1979.

7. Raman, C. V.; Krishnan, K. S.; Nature 1928, 121, 501.

8. Nestor, J. R.; Lippincott, E. R.; J. Raman Spectrosc. 1973, 1, 305.

9. Faria, D. L. A.; Temperini, M. L. A.; Sala, O.; Quim. Nova 1999, 22, 541

10. Campion, A.; Kambhampati, P.; Chem. Soc. Rev. 1998, 27, 241.

11. Norrod, K. L.; Sudnik, L. M.; Rousell, D.; Rowlen, K. L.; Appl. Spectrosc. 1997, 51, 994

12. Pinheiro, L. S.; Temperini, M. L. A.; J. Electroanal. Chem. 1990, 295, 169; Noda, L. K.; Sala, O.; J. Mol. Struct. 1987, 4, 11; Rubim, J. C.; J. Electroanal. Chem. 1987, 220, 339.

13. Vo-Dinh, T.; Em Photonic Probes of Surfaces; Halevi, P., ed.; Elsevier: New York, 1995, p. 65; Alak, A. M.; Vo-Dinh T.; Anal. Chem. 1987, 59, 2149; Alak, A. M.; Vo-Dinh, T.; Anal. Chim. Acta 1988, 206, 333; Narayanan V. A.; Begun, G. M.; Stokes, D. L.; Sutherland, W. S.; Vo-Dinh, T.; J. Raman Spectrosc. 1992, 23, 281.

14. Nabiev, I.; Chourpa, I.; Manfait, M.; J. Raman Spectrosc. 1994, 25, 13.

15. Kneipp, K.; Haka, A. S.; Kneipp, H.; Badizadegan, K.; Yoshizawa, N.; Boone, C.; Shafer-Peltier; K. E.; Motz, J. T.; Dasari, R. R.; Feld, M. S.; Appl. Spectrosc. 2002, 56, 150 .

16. Kneipp, K.; Wang, Y.; Kneipp, H.; Perelman, L. T.; Itzkan, I.; Dasari, R.; Feld, M. S.; Phys. Rev. Lett. 1997, 78, 1667.

17. Nie, S. M.; Emery, S. R.; Science 1997, 275, 1102.

18. Kneipp, K.; Kneipp, H.; Itzan, I.; Dasari, R.; Feld, M. S.; Chem. Rev. 1999, 99, 2957.

19. Li, Y. S.; Wang, Y.; Appl. Spectrosc. 1992, 46, 142 ; Szabo, N. J.; Winefordner, J.D.; Appl. Spectrosc. 1997, 51, 965 ; Szabo, N. J.; Winefordner, J. D.; Appl. Spectrosc. 1998, 52, 500 ; Polwart, E.; Keir, R. L.; Davidson, C. M.; Smith, W. E.; Sadler, D. A.; Appl. Spectrosc. 2000, 54, 522; Dijkstra, R. J.; Gerssen, A.; Efremov, E. V.; Ariese, F.; Brinkman, U. A. T.; Gooijer, C.; Anal. Chim. Acta 2004, 508, 127 ; Wei, G.; Zhou, H.; Liu, Z.; Li, Z.; Appl. Surf. Sci. 2005, 240, 260.

20. Lacy, W. B.; Olson, L. G.; Harris, J. M.; Anal. Chem. 1999, 71, 2564; Jones, J. C.; McLaughlin, C.; Littlejohn, D.; Sadles, D. A.; Graham, D.; Smith, W. E.; Anal. Chem. 1999, 71, 596.

21. Creighton, J. A.; Blatchford, C. G.; Albrecht, M. G.; J. Chem. Soc., Faraday Trans. II 1979, 75, 790.

22. Lee, P. C.; Meisel, D.; J. Phys. Chem. 1982, 86, 3391.

23. Leopold, N.; Lendl, B. ; J. Phys. Chem. 2003, 107, 5723.

24. Fleischmann, M.; Hendra, P. J.; McQuillan, A. J. ; Chem. Phys. Lett. 1974, 26, 163.

25. Li, W.-H.; Li, X.-Y.; Yu, N.-T. ; Chem. Phys. Lett. 1999, 312, 28.

26. Burstein, E.; Burns, G.; Dacol, F.H. ; Solid State Commun. 1983, 46, 595. 